$92 \mathrm{~nm})$ in SSc compared to OA $(\mathrm{p}<0.05)$ and in the range of MV (230$510 \mathrm{~nm}$ ) (figure below). Finally, we observed a positive association between HLA-G+ with Erythrocyte Sedimentation Rate (ESR) ( $\beta=0.34$; $\mathrm{p}<0.01)$.

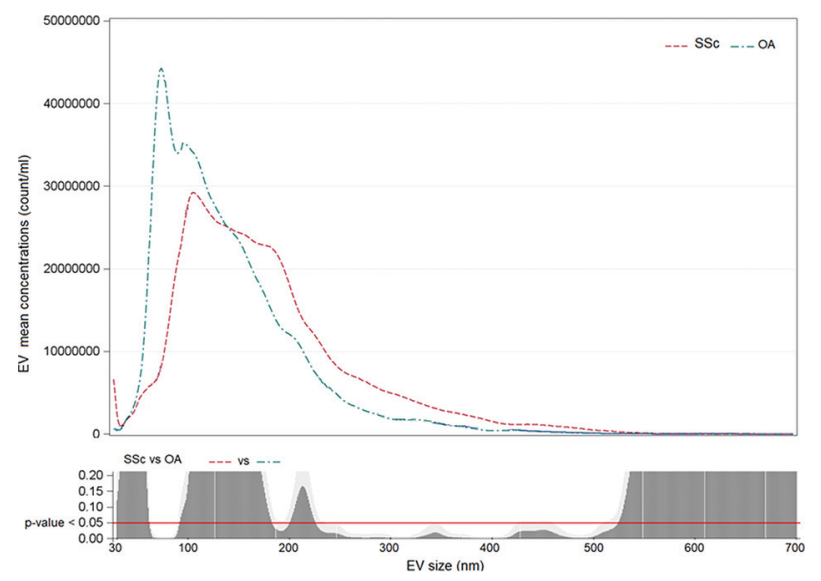

Abstract THU0344 - Figure 1

Conclusion: In our study, limited cutaneous SSc showed different EVs concentrations from controls: SSc tends to have less exosomes and more MV than OA. Moreover, environmental stimuli (e.g. PM exposure) are confirmed to be able to influence HERV-w+ MV release both in SSc and controls. Finally, in SSc patients PM exposure could significantly alter the release of HLA-G+ MV that has been correlated to the process of self-tolerance maintenance. Further studies are required to fully unlock the role of $\mathrm{PM}$ and $\mathrm{EVS}$ in SSc.

Disclosure of Interests: None declared

DOI: 10.1136/annrheumdis-2019-eular.3243

\section{THU0345 TEXTURE-BASED RADIOMICS FEATURES DISCRIMINATE DIFFERENT STAGES OF EXPERIMENTAL INTERSTITIAL LUNG DISEASE}

Janine Schniering ${ }^{1}$, Matthias Brunner ${ }^{1}$, Oliver Distler ${ }^{1}$, Matthias Guckenberger ${ }^{2}$, Marta Bogowicz ${ }^{2}$, Diem Vuong ${ }^{2}$, Cristina Müller ${ }^{3}$, Thomas Frauenfelder ${ }^{4}$, Stephanie Tanadini-Lang ${ }^{2}$, Britta Maurer ${ }^{1} .{ }^{1}$ Center of Experimental Rheumatology, Department of Rheumatology, University Hospital Zurich, Zurich, Switzerland; ${ }^{2}$ Department of Radiation Oncology, University Hospital Zurich, Zurich, Switzerland; ${ }^{3}$ Center for Radiopharmaceutical Sciences, Paul Scherrer Institute, Villigen-PSI, Switzerland; ${ }^{4}$ Institute of Diagnostic and Interventional Radiology, University Hospital Zurich, Zurich, Switzerland

Background: Interstitial lung disease (ILD) is a life-threating complication of systemic sclerosis (SSc). There is an unmet need for validated, routinely available biomarkers for disease staging and individualized patient stratification. In that respect, high-resolution computed tomography (HRCT), routinely performed in the work-up of SSc-ILD patients, has great potential as a source for non-invasive imaging biomarkers. Recently, radiomics, the quantitative extraction of hundreds of radiologic image features, has emerged as a powerful tool for biomarker research and precision medicine approaches; however, so far, it has been applied predominantly in oncology.

Objectives: To evaluate the performance of CT-based radiomics for disease detection, staging and as surrogate marker for tissue pathophysiology in the model of bleomycin (BLM)-induced lung fibrosis.

Methods: To mimic different stages of SSc-ILD, small-animal CT imaging was performed at days 3,7 , and 14 after instillation of BLM or saline solution, respectively ( $n=5-6$ mice/group). Trained observers manually segmented the left and right mouse lungs in the CT images, and 154 radiomics features were extracted including 17 histogram- and 137 texturebased features using the in-house developed software Z-Rad (Python 2.7). To link the radiomics features with the underlying pathophysiology, Pearson correlation analysis between radiomics and histological features (Ashcroft score, Sirius red and CD45 staining positivity) was performed. Results: CT imaging visualized morphological changes of the lung architecture in BLM-treated mice, as evidenced by a time-dependent increase of grey areas on chest CT images and a gradual increase in tissue density on density-masked lungs. Accordingly, 6 out of 17 classical histogram features (e.g. mean CT density, skewness and kurtosis) precisely distinguished diseased from healthy mice with area under the curve values (AUCs) of $>0.87 \quad(p<0.05)$ and thus detected lung remodelling. However, histogram-based features failed to differentiate between the different time points and thus stages of experimental lung fibrosis as investigated by receiver operating characteristic curve analysis and by hierarchical clustering. In contrast, 59 out of 137 texture-based radiomics features, including features describing image homogeneity or contrast detected ILD and distinguished the different stages with excellent accuracy (AUCs $>0.84$ $\mathrm{p}<0.05)$ thus outperforming the classical histogram features.

In addition, texture-based, but not histogram-based features were associated $\left(R^{2}>0.5, \quad p<0.05\right)$ with histological parameters, thereby possibly reflecting changes in lung (micro-)architecture, which may result from both inflammatory and/or fibrotic changes.

Conclusion: If confirmed in human SSc-ILD, texture-based radiomics features have great potential to provide biological and stage-specific quantitative information on lung (micro-)architecture and thus could serve as quantitative imaging biomarkers for guided decision making in SSc-ILD.

Disclosure of Interests: : Janine Schniering: None declared, Matthias Brunner: None declared, Oliver Distler Grant/research support from: Prof Distler received research funding from Actelion, Bayer, Boehringer Ingelheim and Mitsubishi Tanabe to investigate potential treatments of scleroderma and its complications, Consultant for: Prof. Distler has/had consultancy relationship within the last 3 years with Actelion, AnaMar, Bayer, Boehringer Ingelheim, ChemomAb, espeRare foundation, Genentech/Roche, GSK, Inventiva, Italfarmaco, iQvia, Lilly, medac, Medlmmune, Mitsubishi Tanabe Pharma, Pharmacyclics, Novartis, Pfizer, Sanofi, Serodapharm and UCB in the area of potential treatments of scleroderma and its complications. In addition, he had/has consultancy relationship within the last 3 years with A. Menarini, Amgen, Abbvie, GSK, Mepha, MSD, Pfizer and UCB in the field of arthritides and related disorders, Matthias Guckenberger Grant/research support from: Grant/research support by Varian Medical Systems and Siemens Healthcare, Marta Bogowicz: None declared, Diem Vuong: None declared, Cristina Müller: None declared, Thomas Frauenfelder: None declared, Stephanie Tanadini-Lang Grant/ research support from: Grant/research support by Varian Medical Systems and Siemens Healthcare, Britta Maurer Grant/research support from: Grant/research support from AbbVie, Protagen, Novartis; congress support from MSD, Pfizer, Roche, and Actelion

DOI: 10.1136/annrheumdis-2019-eular.809

\section{THU0346 EVALUATION OF A NOVEL PARTICLE-BASED ASSAY FOR DETECTION OF MYOSITIS SPECIFIC ANTIBODIES}

Michael Mahler ${ }^{1}$, Kishore Malyavantham ${ }^{1}$, Michaelin Richards ${ }^{1}$, Chelsea Bentow ${ }^{1}$, Silvia Casas ${ }^{1}$, Eva Balada ${ }^{2}$, Maite Sanz ${ }^{3}$, M. Angeles Martinez ${ }^{4}$, Albert SelvaO'callaghan ${ }^{3}$. ${ }^{1}$ Inova Diagnostics, RandD, San Diego, United States of America; ${ }^{2}$ Hospital Vall d'Hebron, Department of Internal Medicine, Barcelona, Spain; ${ }^{3}$ Hospital Vall d'Hebron, Immunology Department, Barcelona, Spain; ${ }^{4}$ Hospital de La Santa Creu i Sant Pau, Immunology Department, Barcelona, Spain

Background: Myositis specific antibodies (MSA) represent important diagnostic tools and also help stratify idiopathic inflammatory myositis (IIM) patients with particular clinical features, treatment responses, and disease outcomes. Standardization of MSA detection is of high importance because these antibodies also have the potential to be used in classification criteria.

Objectives: The objective of this study was to evaluate the clinical performance of a novel particle based multi-analyte technology (PMAT) for the detection of MSA as an aid in the diagnosis and also in the differentiation of IIM subtypes.

Methods: The study included 464 patient samples collected at Hospital Vall d'Hebron, Autonomous University of Barcelona, most of whom had a diagnosis of IIM $(n=264)$. As controls, samples from patients with myositis like conditions ( $M L, n=20)$, rheumatoid arthritis ( $R A, n=33$ ), systemic lupus erythematosus (SLE, $n=40$ ), Sjögren's syndrome (SjS, $n=25)$, infectious diseases $(I D, n=40)$ and healthy individuals $(H I, n=42)$ were included. All samples were tested using a novel fully automated particle-based multianalyte technology (PMAT, Inova Diagnostics, research use only; Jo-1 PL-7, PL-12, EJ, Mi-2b, NXP2, SAE, TIF1y, MDA5, HMGCR, SRP) which utilizes paramagnetic particles with unique signatures and a digital interpretation system 
Abstract THU0346 -Table 1.

\begin{tabular}{|l|l|l|l|l|}
\hline Analyte & IIM Subtype & Sensitivity $(95 \% \mathrm{Cl})$ & Specificity $(95 \% \mathrm{Cl})$ & Odds Ratio $(95 \% \mathrm{Cl})$ \\
\hline Jo-1 & ASS $(\mathrm{n}=67)$ & $71.6 \%(59.9-81.0 \%)$ & $98.0 \%(94.9-99.2 \%)$ & $121.9(40.8-360.0)$ \\
\hline PL-7 & ASS & $10.4 \%(5.2-20.0 \%)$ & $100.0 \%(98.1-100.0 \%)$ & $+\infty(5.8-+\infty)$ \\
\hline PL-12 & ASS & $7.5 \%(3.2-16.3 \%)$ & $100.0 \%(98.1-100.0 \%)$ & $+\infty(4.0-+\infty)$ \\
\hline EJ & ASS & $3.0 \%(0.8-10.2 \%)$ & $99.5 \%(97.2-99.9 \%)$ & $6.0(0.7-46.8)$ \\
\hline Mi-2 & DM $(n=70)$ & $21.4 \%(13.4-32.4 \%)$ & $97.9 \%(94.8-99.2 \%)$ & $13.0(4.3-38.7)$ \\
\hline NXP2 & DM & $7.1 \%(3.1-15.7 \%)$ & $98.5 \%(95.6-99.5 \%)$ & $4.9(1.3-19.1)$ \\
\hline SAE & DM & $11.4 \%(5.9-21.0 \%)$ & $99.0 \%(96.3-99.7 \%)$ & $12.4(2.9-52.9)$ \\
\hline TIF1y & DM & $15.7 \%(9.0-26.0 \%)$ & $96.2 \%(92.0-98.3 \%)$ & $4.8(1.7-13.0)$ \\
\hline TIF1y & CDM $(n=35)$ & $62.9 \%(46.3-76.8 \%)$ & $96.2 \%(92.0-98.3 \%)$ & $43.2(15.1-122.8)$ \\
\hline MDA5 & CADM $(\mathrm{n}=21)$ & $71.4 \%(50.0-86.2 \%)$ & $97.1 \%(94.2-98.6 \%)$ & $84.3(25.7-277.5)$ \\
\hline HMGCR & IMNM $(\mathrm{n}=16)$ & $50.0 \%(28.0-72.0 \%)$ & $96.8 \%(93.8-98.4 \%)$ & $30.0(9.2-98.2)$ \\
\hline
\end{tabular}

ASS = Anti-Synthetase Syndrome, DM = Dermatomyositis, $C D M=$ Cancer Associated Dermatomyositis, CADM = Amyopathic Dermatomyositis

Results: The sensitivity/specificity of the individual MSA were: $19.7 \% /$ 100\% (Jo-1), $\quad 7.2 \% / 100.0 \% \quad$ (Mi-2), $3.0 \% / 99.0 \% \quad$ (NXP2), $3.8 \% / 100.0 \%$ (SAE), 2.7\%/100.0\% (PL-7), 1.9\%/99.5 (PL-12), 1.1\%/100.0\% (EJ), 15.5\%/ $99.5 \%$ (TIF1y), 8.3\%/98.5\% (MDA5), 6.1\%/99.0\% (HMGCR) and $1.9 \% /$ $98.5 \%$ (SRP). The overall clinical performance was: sensitivity $68.2 \%$ (95\% confidence interval $62.3-73.5 \%)$, specificity $94.0 \% \quad(95 \% \mathrm{Cl} \quad 89.8-$ $96.5 \%$ ) and odds ratio 33.8 . In the table below, the sensitivity and specificity of each analyte for IIM subtypes was calculated along with odds ratio.

Conclusion: The novel PMAT used to detect a spectrum of MSA in IIM on a fully automated system showed good sensitivity and specificity in line with the known associations of MSA. Sensitivities and specificities of the individual MSA are within expected ranges. Lastly, the individual markers help to stratify patients into IIM subtype which is important for management of the patients.

Disclosure of Interests: Michael Mahler Employee of: Inova Diagnostics (Not pharmaceutical, diagnostics company), Kishore Malyavantham Employee of: Inova Diagnostics, Michaelin Richards Employee of: Inova Diagnostics, Chelsea Bentow Employee of: INOVA Diagnostics, Silvia Casas Employee of: Inova Diagnostics, Eva Balada: None declared, Maite Sanz: None declared, M. Angeles Martinez: None declared, Albert SelvaO'Callaghan: None declared

DOI: 10.1136/annrheumdis-2019-eular.8101

\section{THU0347 TESTING THE IN VITRO EFFECTS OF NINTEDANIB ON CIRCULATING FIBROCYTES AND RESIDENT SKIN FIBROBLASTS FROM THE SAME SYSTEMIC SCLEROSIS PATIENTS: PRELIMINARY RESULTS}

Maurizio Cutolo ${ }^{1}$, Paola Contini ${ }^{2}$, Giulia Martinelli ${ }^{1}$, Paola Montagna ${ }^{1}$, Samuele Tardito ${ }^{1}$, Sabrina Paolino ${ }^{1}$, Massimo Patanè ${ }^{1}$, Carmen Pizzorni ${ }^{1}$, Elisa Alessandri ${ }^{1}$, Veronica Tomatis ${ }^{1}$, Vanessa Smith ${ }^{3,4,5}$, Stefano Soldano ${ }^{1}$ ${ }^{1}$ University of Genova, IRCCS Polyclinic San Martino Hospital, Research Laboratory and Academic Division of Clinical Rheumatology, Dept. of Internal Medicine, Genoa, Italy, ${ }^{2}$ University of Genova, IRCCS Polyclinic San Martino Hospital, 2 Clinical Immunology, Dept. of Internal Medicine, Genoa, Italy; ${ }^{3}$ Ghent University Hospital, Dept. of Rheumatology, Ghent University Hospital, Ghent, Belgium; Dept. of Internal Medicine, Ghent, Belgium; ${ }^{4}$ Ghent University, Dept. of Internal Medicine, Ghent, Belgium; ${ }^{5}$ VIB Inflammation Research Center (IRC), Unit for Molecular Immunology and Inflammation, Ghent, Belgium

Background: The fibrosis in systemic sclerosis (SSc) progresses from microvascular alterations, immune system activation, and increased extracellular matrix protein synthesis into the skin and internal organs, primarily mediated by myofibroblasts. ${ }^{1}$ Myofibroblasts are characterized by a higher expression of $\alpha$-smooth muscle actin $(\alpha S M A)$ and by the overproduction of type I collagen (COL1) and fibronectin (FN). ${ }^{2}$ Although myofibroblasts primarily derive from fibroblasts differentiation and transition, circulating fibrocytes represent a further important source. ${ }^{3}$ Nintedanib is a tyrosine kinase inhibitor that interacts with several inflammatory and profibrotic pathways implicated in the pathogenesis of fibrosis, including those of platelet derived growth factor receptors, vascular endothelial growth factor receptors and fibroblast growth factor receptors. ${ }^{4}$

Objectives: To investigate, in primary cultures, the effects of nintedanib on the differentiation of circulating fibrocytes and on the profibrotic activity of skin fibroblasts isolated from the same SSc patients.

Methods: Circulating fibrocytes and fibroblasts were obtained from peripheral blood and skin biopsies, respectively, from three untreated SSc patients with diffuse skin involvement (mean age $55 \pm 6 \mathrm{yrs}$ ). To investigate the complete differentiation, fibrocytes were maintained in DMEM at $20 \%$ of foetal bovine serum, either with or without treatment with nintedanib at the concentrations of $0.1 \mu \mathrm{M}$ and $1 \mu \mathrm{M}$ for 8 days. ${ }^{5}$ Fibrocytes were characterized as $\mathrm{CD} 45^{+} \mathrm{CXCR} 4^{+} \mathrm{COL} 1^{+}$cells, and their percentage was detected by Flow Cytometry analysis. ${ }^{6}$ Fibroblasts were grown until the $3^{\text {dr }}$ culture passage and then maintained in normal growth medium with or without nintedanib treatment for 4,24 and 48 hours. The gene expressions of $\alpha$ SMA, COL1 and FN were evaluated by qRT-PCR.

Results: Nintedanib reduced the percentage of differentiated SSc fibrocytes $\left(\mathrm{CD} 45^{+} \mathrm{CXCR} 4^{+} \mathrm{COL} 1^{+}\right.$cells), already at the concentration of $0.1 \mu \mathrm{M}$ compared with untreated fibrocytes. In cultured SSc skin fibroblasts, nintedanib $1 \mu \mathrm{M}$ downregulated $\alpha \mathrm{SMA}, \mathrm{COL} 1$ and $\mathrm{FN}$ expressions already after 4 hours of treatment, and this effect was also maintained after 24- and 48-hours' treatment. Nintedanib $0.1 \mu \mathrm{M}$ downregulated the $\alpha \mathrm{SMA}$ expression at all timepoints, whereas the downregulation of COL1 and FN expressions was observed at 4 and 24 hours, with no more effect at 48 hours of treatment compared with untreated cells.

Conclusion: Initial experiments seem to indicate an evident in vitro concentration-dependent antifibrotic activity of nintedanib on SSc fibrocytes and skin fibroblasts from the same SSc patients; in particular by reducing the fibrocyte differentiation as potential source of myofibroblasts and con trasting the profibrotic activity of already activated fibroblasts/myofibroblasts. These results have translational implications in clinical trials using nintedanib in SSc patients.

\section{REFERENCES}

[1] Furue M, et al. Immunol Res 2017;65:790-7.

[2] Kendall RT, et al. Front Pharmacol 2014;5:123

[3] Strieter RB, et al. J Leukocyte Biol 2009;88:111-8.

[4] Huang J, et al. Ann Rheum Dis 2017;76:1941-8.

[5] Cutolo M, et al. Arthritis Res Ther 2018;20:157.

Disclosure of Interests: None declared

DOI: 10.1136/annrheumdis-2019-eular.6909

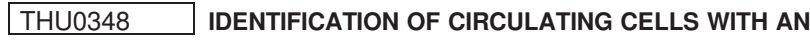 HYBRID M1/M2 MACROPHAGE PHENOTYPE IN SYSTEMIC SCLEROSIS PATIENTS AND CORRELATIONS WITH SELECTED CLINICAL ASPECTS}

Stefano Soldano ${ }^{1}$, Amelia Chiara Trombetta ${ }^{1}$, Paola Contini $^{2}$, Greta Pacini ${ }^{1}$, Veronica Tomatis ${ }^{1}$, Paola Montagna ${ }^{1}$, Massimo Patanè ${ }^{1}$, Elisa Alessandri ${ }^{1}$, Federica Goegan 1 , Vanessa Smith ${ }^{3,4}$, Maurizio Cutolo'. ' ${ }^{1}$ University of Genova, IRCCS Polyclinic San Martino Hospital, Research Laboratory and Academic Division of Clinical Rheumatology, Dept. of Internal Medicine, Genoa, Italy, ${ }^{2}$ University of Genova, IRCCS Polyclinic San Martino Hospital, Clinical Immunology, Dept. of Internal Medicine, Genoa, Italy; ${ }^{3}$ Ghent University, Ghent, Dept. of Rheumatology, Ghent University Hospital, Ghent, Belgium; Dept. of Internal Medicine, Ghent, Belgium; ${ }^{4}$ VIB Inflammation Research Center (IRC), Unit for Molecular Immunology and Inflammation, Ghent, Belgium

Background: Systemic sclerosis (SSc) is characterized by immune system alterations, vascular damage and fibrosis (1). Macrophages seem to have an emerging role in SSc, and the characterization of their polarized phenotype, starting from the dichotomic definition of classically activated (M1) or alternatively activated (M2) macrophages, is a recent research topic of interest (2)

Objectives: The study investigated a possible imbalance in the distribution of circulating cells expressing $\mathrm{M} 1$ and $\mathrm{M} 2$ markers in SSc patients (pts) compared to healthy subjects (HSs), and the presence of circulating cells co-expressing M1 and M2 surface markers. Possible correlations between their percentage and selected SSc clinical aspects were investigated.

Methods: In the study 55 SSc pts (50 females/5 males, mean age 64 $\pm 13 \mathrm{yrs}$ ), fulfilling the new EULAR/ACR criteria for SSc diagnosis, and 27 age-matched HSs (25 females/2 males, mean age $57 \pm 7$ yrs) were enrolled after written informed consent. Nailfold videocapillaroscopy (NVC), evaluation of SSc-related antibodies and pulmonary functional tests were performed. In particular, circulating cells belonging to the leukocyte and monocyte populations (CD $45^{+}$and $\mathrm{CD} 14^{+}$cells) were investigated by flow cytometry (FC) using the surface markers characterizing M1 (CD80 CD86, TLR2, TLR4) and M2 phenotypes (CD204, CD206, CD163). Statistical analysis was performed using Mann-Whitney and Kruskal-Wallis tests, and correlations were explored by bivariate Pearson's analysis.

Results: Increased circulating cells showing an M2 phenotype and characterized as $\mathrm{CD}_{20} 4^{+} \mathrm{CD} 206^{+} \mathrm{CD} 163^{+}$cells was observed in SSc pts compared to HSs $(p<0.0001)$, whereas no difference in CD80 ${ }^{+}$CD86 ${ }^{+} \mathrm{TLR}_{2}{ }^{+} \mathrm{TLR}^{+}{ }^{+}(\mathrm{M} 1)$ cell percentage was observed. A significant higher percentage of circulating cells showing a hybrid M1/M2 phenotype (CD204 ${ }^{+} \mathrm{CD} 206^{+} \mathrm{CD}_{163}{ }^{+} \mathrm{TLR}^{+} \mathrm{CD} 80^{+} \mathrm{CD} 86^{+}$cells) was observed in SSc pts compared to HSs. These hybrid M1/M2 cells were significantly increased in SSc pts either treated with steroids or under no immunosuppressive treatment $(p<0.01 ; p<0.05)$, as well as in Scl70 ${ }^{+} p t s \quad(p<0.05$ vs. Scl70 $p t s)$ 